\title{
Comparison of the acute effects of pacing the atrial septum, right atrial appendage, coronary sinus os, and the latter two sites simultaneously on the duration of atrial activation
}

\author{
D H Bennett
}

\begin{abstract} os, and dual site pacing on the duration of atrial activation. site stimulation. and 98 (28) ms for coronary sinus os pacing. associated with the latter pacing mode.

(Heart 2000;84:193-196)

Both simultaneous stimulation of the right atrial appendage and the coronary sinus os-that is, dual site pacing - and right atrial septal pacing have been reported to shorten the duration of atrial activation and thereby prevent or diminish the frequency of paroxysmal atrial fibrillation. ${ }^{12}$ Comparisons of the effect on atrial activation by pacing at different atrial sites have been reported but did not include atrial septal stimulation. ${ }^{34}$ In this report, the acute effects on the duration of atrial activation of atrial septal, coronary sinus os, right atrial appendage, and dual site pacing are compared.
\end{abstract}

Objective-To compare the acute effects of right atrial appendage, atrial septal, coronary sinus

Methods-20 patients with a variety of cardiac conditions underwent an intracardiac electrophysiological study. Electrograms were recorded from the right atrial appendage and at multiple sites within the coronary sinus. The duration of atrial activation was measured during pacing at the right atrial appendage, atrial septum, and coronary sinus os, and also during dual

Results-The duration of atrial activation with atrial appendage pacing was notably longer $(p<0.001)$ than with dual site, septal, or coronary sinus os pacing, but there were no significant differences in atrial activation times between these latter three pacing modes. When stimulating the atria at a cycle length of $500 \mathrm{~ms}$, the mean (SD) duration of atrial activation was 145 (37) ms for right atrial appendage pacing, 93 (26) $\mathrm{ms}$ for dual site pacing, 96 (28) ms for septal pacing,

Conclusions-Assuming that the duration of atrial activation is an important determinant of predisposition to paroxysmal atrial fibrillation, atrial septal pacing or coronary sinus os pacing would appear to offer the same advantage as dual site pacing without the additional complexities

Keywords: atrial septal pacing; dual site pacing; atrial activation; atrial fibrillation

\section{Methods}

Nine male and 11 female patients were studied. They were undergoing intracardiac electrophysiological testing for clinical purposes. Their ages ranged from 27-72 years (mean (SD), 48 (13)). All gave informed consent.

Sixteen of the patients underwent radiofrequency ablation: four had accessory atrioventricular pathways, five had atrioventricular nodal re-entrant tachycardia, three had atrial flutter, one had right ventricular outflow tract tachycardia, and three underwent atrioventricular nodal ablation for paroxysmal atrial fibrillation or tachycardia. Atrial activation durations were measured in the few minutes after successful ablation, while the patient was being observed to ensure that the ablated pathway or circuit did not recover.
In addition, four patients - three with paroxysmal atrial fibrillation and one with paroxysmal atrial tachycardia-underwent atrial stimulation studies immediately before implantation of a dual chamber pacemaker.

After withdrawal of antiarrhythmic treatment, each patient underwent an electrophysiological study under local anaesthesia with oral or intravenous sedation. As part of the clinical procedure, at least three electrodes were advanced to the heart percutaneously through the right femoral vein in each patient. A quadripolar electrode was advanced to the right atrial appendage for stimulation and recording. A deflectable, steerable quadripolar electrode was used for mapping and, where required, for delivery of radiofrequency energy. A 2 French 16 pole electrode (Cardima Medlink Europe, Nieuwegein, The Netherlands) was advanced as far as possible into the coronary sinus through a 6 French Amplatz guiding catheter (fig 1), except in those patients undergoing ablation for atrial flutter where a 7 French duodecapolar electrode (Daig Corp, Minnetonka, Minnesota, USA) was positioned in the right atrium with its distal end in the coronary sinus os.

Surface and intracardiac bipolar electrograms were recorded using a computerised multichannel system (EPMedSystems, Costa Mesa, California, USA) which also facilitated delivery of pacing stimuli (fig 2).

Pacing stimuli were delivered at $600 \mathrm{~ms}$ and then at $500 \mathrm{~ms}$ cycle lengths in turn to the right 


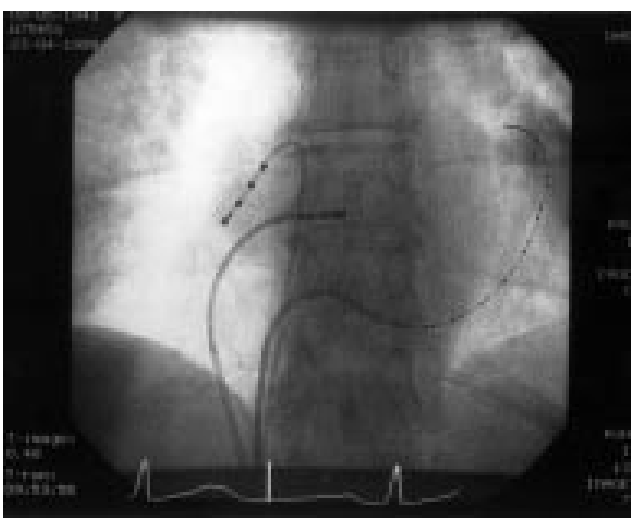

Figure 1 Anteroposterior radiograph showing positions of the multipolar electrode in the coronary sinus and quadripolar electrodes in the right atrial appendage and on the atrial septum.

atrial appendage, coronary sinus os, atrial septum, and simultaneously to the coronary sinus os and right atrial appendage. To ensure effective dual site pacing, the poles of the atrial appendage lead and then the coronary sinus os lead were in turn briefly disconnected to make certain that atrial pacing continued through the lead which remained connected. In the 17 patients in whom an Amplatz shape catheter was introduced into the coronary sinus, the os was identified by injection of contrast medium. In the patients undergoing ablation for atrial flutter, the tip of the duodecapolar electrode was advanced to the distal coronary sinus after successful isthmus ablation.

The duration of atrial activation was measured as the interval between delivery of the pacing stimulus and subsequent inscription of the latest atrial electrogram, whether recorded from the coronary sinus or the right atrium.

Atrioventricular conduction time was measured during each atrial pacing mode.

Right and left atrial synchronisation was measured as the interval between inscription of

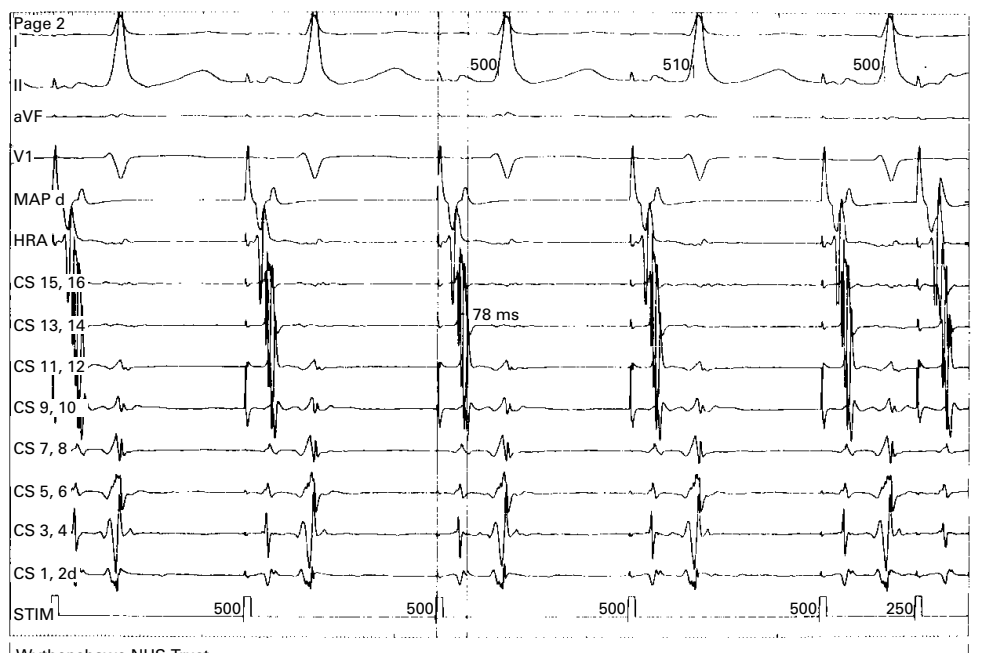

Figure 2 Surface and intracardiac electrograms during atrial septal pacing. The interval between pacing stimulus and latest left atrial electrogram, which is recorded halfway between the most proximal coronary sinus electrode (CS 15,16p) and the most distal (CS $1,2 d)$, is $78 \mathrm{~ms}$. The last cycle follows an atrial premature stimulus and shows the same sequence of atrial activation.
Table 1 Duration of atrial activation (ms) with different atrial pacing modes at $600 \mathrm{~ms}$ cycle length

\begin{tabular}{lllll}
\hline & Appendage & Coronary os & Dual site & Septum \\
\hline Range & 69 to 236 & 57 to 183 & 61 to 154 & 115 to 165 \\
Mean & 144 & 100 & 94 & 96 \\
SD & 40 & 28 & 23 & 27 \\
CI & 21 & 15 & 12 & 14 \\
Number of & & & 17 & 17 \\
$\quad$ patients & 17 & 17 & 17
\end{tabular}

$\mathrm{SD}$, standard deviation; CI, confidence interval.

Table 2 Duration of atrial activation (ms) with different atrial pacing modes at $500 \mathrm{~ms}$ cycle length

\begin{tabular}{lllll}
\hline & Appendage & Coronary os & Dual site & Septum \\
\hline Range & 86 to 244 & 57 to 183 & 62 to 164 & 46 to 175 \\
Mean & 145 & 98 & 93 & 96 \\
SD & 37 & 30 & 26 & 28 \\
CI & 17 & 14 & 12 & 13 \\
Number of & & & & \\
$\quad$ patients & 20 & 20 & 20 & 20 \\
\hline
\end{tabular}

the electrogram recorded from the right atrial appendage and latest coronary sinus electrogram. ${ }^{2}$

\section{Results}

The duration of atrial activation with atrial appendage pacing was notably longer $(p<0.001)$ than during dual site, septal, or coronary sinus os pacing, but there were no significant differences in atrial activation times between these latter three pacing modes (tables 1 and 2). In three patients, the sinus node rate was too high to pace at a cycle length of $600 \mathrm{~ms}$. The two patients with very long activation times had pre-existing interatrial conduction delays and had received intravenous flecainide during the procedure to terminate an episode of atrial fibrillation.

The latest atrial electrogram during atrial pacing was not always recorded in the distal coronary sinus. In 15 patients it was recorded in the mid coronary sinus, and in one patient who had received flecainide and who had very long interatrial conduction times, pacing modes other than right atrial appendage stimulation resulted in the right atrial electrogram following the coronary sinus electrograms.

Atrioventricular conduction was measured during the different atrial pacing modes at a cycle length of $600 \mathrm{~ms}$ in 13 patients (table 3). Clearly, it could not be measured in the three patients who had undergone atrioventricular nodal ablation or, at that cycle length, in the three patients with a fast sinus node rate. Right atrial appendage pacing led to slightly longer atrioventricular conduction than the other pacing modes $(\mathrm{p}<0.001)$.

Table 3 Duration of atrioventricular conduction (ms) with different atrial pacing modes at $600 \mathrm{~ms}$ cycle length

\begin{tabular}{lllll}
\hline & Appendage & Coronary os & Dual site & Septum \\
\hline Range & 136 to 330 & 80 to 243 & 82 to 151 & 100 to 237 \\
Mean & 208 & 172 & 174 & 164 \\
SD & 59 & 50 & 53 & 48 \\
CI & 35 & 30 & 32 & 28 \\
Number of & & 13 & 13 & 13 \\
$\quad$ patients & 13 & 13 & & \\
\hline
\end{tabular}


Table 4 Measure of atrial synchronisation (ms)

\begin{tabular}{lll}
\hline & Sinus rhythm & Atrial septal pacing \\
\hline Range & 40 to 124 & 20 to 82 \\
Mean & 78 & 51 \\
SD & 26 & 25 \\
CI & 14 & 14 \\
Number of patients & 14 & 14 \\
\hline
\end{tabular}

Measures of right and left atrial synchronisation are given in table 4.

\section{Discussion}

Several factors may predispose to paroxysmal atrial fibrillation, including bradycardia, autonomic nervous system activity, delayed intraatrial conduction, atrial mass, atrial stretch, and atrial ectopic beats. ${ }^{5}$ A major factor is dispersion of atrial refractoriness. This can be lessened by reducing the duration of atrial activation. ${ }^{5}$ Hence an objective in pacing for the prevention of atrial fibrillation is to shorten atrial activation and thereby the dispersion of atrial refractoriness. ${ }^{125}$ The conventional site for atrial pacing is the right atrial appendage, but stimulation at this site can lead to delayed intra-atrial and interatrial conduction. ${ }^{2}$

In this study, I compared atrial activation during right atrial appendage, atrial septal, coronary sinus os, and dual site pacing. The duration of atrial activation with atrial appendage pacing was notably longer than during dual site, septal, or coronary sinus os pacing, but there were no significant differences in atrial activation times between these latter three pacing modes.

The duration of atrial activation was measured as the interval between delivery of the pacing stimulus and subsequent inscription of the latest intracardiac atrial electrogram, whether recorded from the coronary sinus or the right atrium. It could be argued that the duration of atrial activation would be better measured from the P wave on a surface ECG, because there could be sites in the atria that would be activated earlier or later than sites where intracardiac electrodes had been positioned. In fact, the earliest and latest intracardiac electrograms did appear to coincide with the beginning and end of the $\mathrm{P}$ wave. However, in contrast to an intracardiac atrial electrogram which has a rapid upstroke, it is difficult to identify the precise point at which a $\mathrm{P}$ wave starts and terminates.

In spite of the heterogeneity of the study group, there were similar findings in all patients. It would seem reasonable, therefore, to assume that the observations in this study are pertinent to patients with atrial fibrillation.

It is not surprising that pacing at sites between the right and left atria leads to shorter durations of atrial activation than pacing the right atrial appendage, and it would be reasonable to hypothesise that the acute data from this study translate to long term pacing. If so, the results suggest that the right atrial appendage should if possible be avoided as a pacing site in patients prone to paroxysmal atrial fibrillation.

If the duration of atrial activation is a major predisposing factor to atrial fibrillation, then it is likely to make little difference whether dual site or single site pacing (atrial septal or coronary sinus os) is employed. Single site pacing does have the advantage of simplicity: dual site pacing requires an additional pacing lead and the use of a special pulse generator or an adapter for connecting two atrial leads. Conversely, Delfaut and colleagues, ${ }^{1}$ in a study of 30 patients, showed that dual site pacing was more effective than right atrial appendage or coronary sinus os pacing, and that the latter mode was not superior to right atrial appendage pacing; however, atrial septal pacing was not studied. Clearly, large randomised studies of long term pacing are required.

If single site atrial pacing is to be pursued, other sites have also been suggested. Papageorgiou and colleagues acquired acute data suggesting that pacing the distal coronary sinus prevents atrial fibrillation ${ }^{6}$; and Padeletti and associates tested the feasibility of pacing Koch's triangle, ${ }^{7}$ showing a pronounced reduction in episodes of atrial fibrillation in patients with sinus bradycardia and atrial fibrillation.

Other studies of different pacing sites examining the duration of atrial activation have assumed that the interval between the right atrial appendage and distal coronary sinus electrograms indicates the duration of atrial activation or interatrial conduction. In the present study, the use of a multipolar electrode in the coronary sinus showed that the latest left atrial electrogram is often recorded in the region of the mid coronary sinus.

Katsivas and colleagues, ${ }^{2}$ in their study of atrial septal pacing, positioned the atrial lead in order to minimise the interval (to $<10 \mathrm{~ms}$ ) between inscription of the electrogram recorded from the right atrial appendage and the latest coronary sinus electrogram. This interval was taken as an indication of right and left atrial synchronisation. In the current study, I found this interval to be substantially longer, though I made no attempt to achieve a short interval between atrial appendage and coronary sinus electrograms when positioning the electrode on the atrial septum.

One possible disadvantage to pacing at sites other than the atrial appendage is inappropriate reduction of the atrioventricular interval. The results of this study did show that atrial appendage pacing resulted in the longest atrioventricular intervals, but the other pacing modes only led to modest shortening of this interval.

CONCLUSIONS

Atrial septal, coronary sinus os, and dual site pacing shorten the duration of atrial activation as compared with right atrial appendage pacing, and may help to deter atrial fibrillation. Assuming that the duration of atrial activation is a major determinant of predisposition to paroxysmal atrial fibrillation, atrial septal or coronary sinus os pacing would appear to offer the same advantage as dual site pacing without the additional complexities associated with the latter pacing mode.

I gratefully acknowledge the help and expertise provided by the physiological measurement technicians. 
1 Delfaut P, Saksena S, Prakash A, et al. Long-term outcome of patients with drug refractory atrial flutter and fibrillation after single- and dual-site right atrial pacing for arrhythmia prevention. $\mathcal{F}$ Am Coll Cardiol 1998 32:1900-8

2 Katsivas A, Manolis AG, Lazaris E, et al. Atrial septal pacing to synchronize atrial depolarisation in patients with delayed interatrial conduction. PACE 1998;21 2220-5.

3 Prakash A, Delfaut P, Krol RB, et al. Regional right and left atrial activation patterns during single- and dual-site atrial pacing in patients with atrial fibrillation. $7 \mathrm{Am}$ Coll Cardiol 1998;82:1197-204.
$4 \mathrm{Yu}$ W, Chen SA, Tai CT, et al. Effects of different atrial pacing modes on atrial electrophysiology: implicating the mechanism of biatrial pacing in prevention of atrial physiology. Circulation 1997;96:2992-6.

5 Ramdat Misier A, Beukema WP, Luttikhuis HA. Multisite or alternate site pacing for the prevention of atrial fibrillation. Am f Cardiol 1999;83:237-40D.

6 Papageorgiou P, Anselme F, Kirchof CJ, et al. Coronary sinus pacing prevents induction of atrial fibrillation. Circulation 1997;6:1893-8.

7 Padeletti L, Porciani MC, Michelucci A, et al. Interatrial septum pacing: a new approach to prevent recurrent atrial fibrillation. F Intervent Cardiac Electrophysiol 1999;3:35-43.

\section{IMAGES IN CARDIOLOGY}

\section{Chronic bilateral carotid body tumours causing carotid sinus hypersensitivity: abolition of symptoms by permanent cardiac pacing}

This ambulatory ECG was recorded from a 59 year old man who had been diagnosed as having nocturnal epilepsy at the age of 26 years.

The patient's initial presentation followed a motorbike accident, although he suffered no head injury. He has subsequently had multiple medical problems and saw an ear, nose, and throat surgeon at the age of 51 because of "sleep apnoea". At that time he was noted to have neck swelling and a computed tomographic (CT) scan (bottom) showed bilateral carotid body tumours, although the diagnosis was not made.

He was referred to a cardiologist because of renewed diagnostic doubt regarding his nocturnal attacks which continued to occur, often in clusters, especially when he lay on his right side, despite anticonvulsant treatment. He gave a history of hypertension, renal dysfunction, and paroxysmal atrial fibrillation.

Following implantation of a permanent pacemaker four years ago he has had no further attacks. When light pressure is applied to the neck masses with the pacemaker's rate limit lowered, asystole is easily produced-carotid sinus hypersensitivity secondary to his carotid body tumours. In the absence of symptoms and because of his other medical problems, surgical removal has not been attempted. The tumours are very slow growing (comparing current CT scans to previous images) and must have produced carotid sinus hypersensitivity even when very small. The likely mechanism for this must be that the tumour changes the pressure transduction mechanisms within the carotid body, leading to hypersensitivity.

MARK TURNER DENIS WILKINS ANDREW J MARSHALI
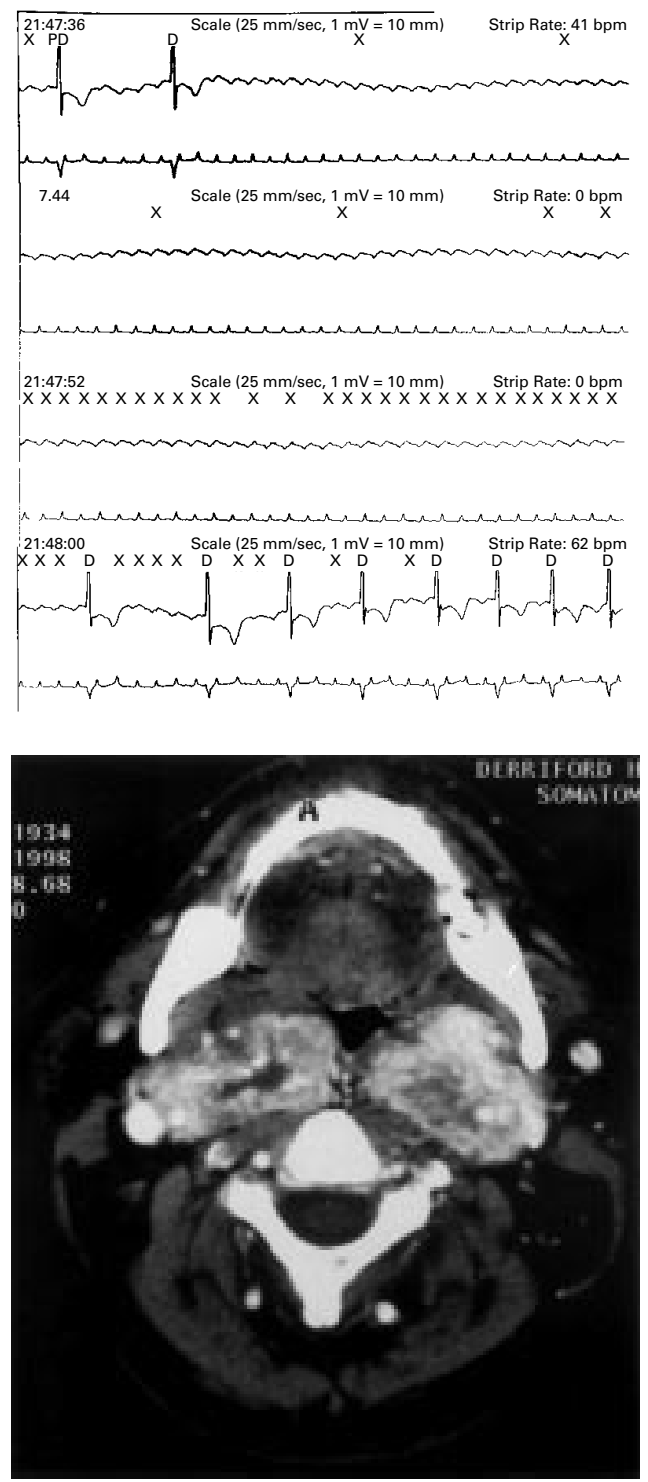